\title{
Endoscopic guide wire gallbladder decompression in a critically ill, septic patient
}

A 57-year-old man with postischemic dilated cardiomyopathy underwent a heart transplant at our center in February 2013. His early postoperative course was complicated by primary graft non-function requiring intensive care treatment.
Six days after transplantation, he developed severe hypoperfusion that required maximal extracorporeal membrane oxygenation (ECMO), placement of an intraaortic balloon pump, and vasoactive drug support.
The patient's bilirubin level increased from $0.8 \mathrm{mg} / \mathrm{dL}$ to $7.5 \mathrm{mg} / \mathrm{dL}$ and his white blood cell (WBC) count from $9 \times 10^{9} / \mathrm{L}$ to $37 \times 10^{9} / \mathrm{L}$. Abdominal ultrasound revealed normal-caliber intrahepatic ducts and a $10-\mathrm{cm}$ sludge-filled gallbladder ( $\bullet$ Fig.1a). A diagnosis of acute biliary septic shock was suspected and an urgent endoscopic retrograde cholangiopancreatography (ERCP) was requested for gallbladder decompression.

A cholangiogram confirmed the normalcaliber intrahepatic ducts and a dilated

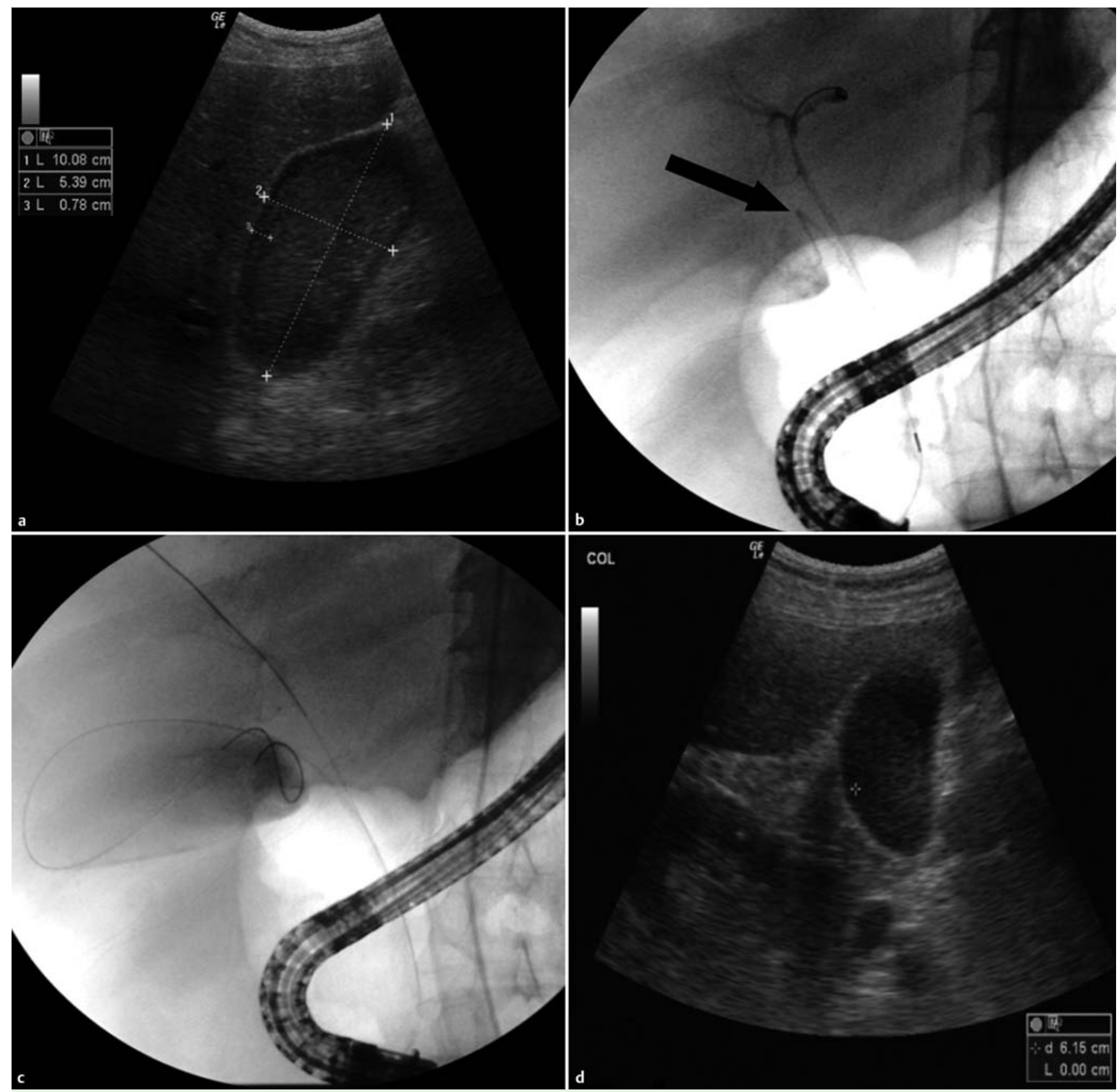

Fig. 1 Images from a 57-year-old man who had received a heart transplant but subsequently developed acute biliary septic shock showing: a a dilated, sludgefilled gallbladder on ultrasound; $\mathbf{b}$ an ultrathin and angulated cystic duct on the cholangiogram, which could not be stented because of its angulation and very small diameter (black arrow); $\mathbf{c}$ the guide wire positioned during endoscopic retrograde cholangiopancreatography (ERCP), which was left in place to decompress the gallbladder; and $\mathbf{d}$ the decompressed gallbladder on ultrasound 48 hours later. 
gallbladder with an ultrathin cystic duct ( Fig. 1 b). After selective cystic duct cannulation, a 0.035-inch guide wire was advanced into the gallbladder. Because of the angulation of the cystic duct and its very small diameter, stenting was not possible. Therefore, in order to straighten the cystic duct and facilitate decompression, the guide wire was left in the gallbladder for 48 hours ( $\bullet$ Fig. 1 c).

During this period, the patient's WBC count dropped to $15 \times 10^{9} / \mathrm{L}$ and the gallbladder size decreased to $6 \mathrm{~cm}$ on ultrasound ( Fig. $1 \mathrm{~d}$ ), while his bilirubin level remained stable. His hemodynamic condition improved, which allowed a reduction in the ECMO and vasoactive support. Unfortunately, 1 week later, the patient developed acute graft rejection and acute respiratory distress syndrome with multiorgan failure, and he died.

Very tight or angulated ducts may be difficult to cannulate with conventional devices. Leaving a guide wire in position for 24 hours to dilate a severe pancreatic duct stricture not traversable with conventional dilators has previously been described [1]. In our case, prolonged guide wire placement provided noninvasive decompression of the gallbladder.

In conclusion, when gallbladder decompression is required in critical "untouchable" patients and especially in the presence of an ultrathin or angulated cystic duct, this technique should be considered before other more invasive procedures, even if its efficacy cannot be fully demonstrated by a single report.

Endoscopy_UCTN_Code_TTT_1AR_2AK

\section{Competing interests: None}

\section{G. Curcio, N. Azzopardi, A. Granata, L. Barresi, I. Tarantino, M. Traina}

Gastroenterology and Endoscopy Unit, Mediterranean Institute for Transplantation and Advanced Specialized Therapies (ISMETT), Palermo, Italy

\section{References}

1 Familiari P, Spada C, Costamagna G. Dilation of a severe pancreatic stricture by using a guidewire left in place for 24 hours. Gastrointest Endosc 2007; 66: 618-620

\section{Bibliography}

DOI http://dx.doi.org/

$10.1055 / \mathrm{s}-0033-1344420$

Endoscopy 2013; 45: E248-E249

(c) Georg Thieme Verlag KG

Stuttgart · New York

ISSN 0013-726X

Corresponding author

G. Curcio, MD

Gastroenterology and Endoscopy Unit

ISMETT, UPMC

Via Tricomi 1

Palermo 90127

Italy

Fax: +39-091-2192400

gcurcio@ismett.edu 